Revue internationale P.M.E.

Économie et gestion de la petite et moyenne entreprise

\title{
Développement et validation d'un instrument de mesure de la qualité dans les entreprises tunisiennes
}

\section{Lassâad Lakhal}

Volume 20, numéro 2, 2007

URI : https://id.erudit.org/iderudit/1008520ar

DOI : https://doi.org/10.7202/1008520ar

Aller au sommaire du numéro

Éditeur(s)

Presses de l’Université du Québec

ISSN

0776-5436 (imprimé)

1918-9699 (numérique)

Découvrir la revue

Citer cette note

Lakhal, L. (2007). Développement et validation d'un instrument de mesure de la qualité dans les entreprises tunisiennes. Revue internationale P.M.E., 20(2), 151-178. https://doi.org/10.7202/1008520ar
Résumé de l'article

Cet article a pour objectif le développement et la validation d'un instrument de mesure de la qualité. La revue de la littérature a permis de retenir 10 pratiques de management de la qualité ainsi que les items qui leur sont liés. L'étude empirique a touché 133 entreprises tunisiennes et a permis, par le biais d'une analyse factorielle confirmatoire, de retenir sept pratiques critiques de management de la qualité et de valider un instrument de mesure. 


\title{
Développement et validation d'un instrument de mesure de la qualité dans les entreprises tunisiennes
}

Lassâad LAKHAL

Faculté de droit et des sciences économiques et politiques de Sousse, Tunisie

\author{
MOTS CLÉS
}

\section{Pratiques de management de la qualité Instrument de mesure de la qualité - Étude empirique}

\begin{abstract}
L'AUTEUR
LASSÂAD LAKHAL est maître assistant à la Faculté de droit et des sciences économiques et politiques de Sousse en Tunisie. II a obtenu son doctorat en gestion auprès de l'Institut supérieur de gestion de Tunis en 2004. II a publié des documents de recherche dans des revues avec comité de lecture, notamment, International Journal of Quality and Reliability Management, Gestion 2000, Revue tunisienne des sciences de gestion, Direction et gestion et la Revue des sciences de Gestion. Adresse: 11, rue de la Libération, cité Ettaamir, 4002, Sousse, Tunisie. Courriel: <lassaad_lakhal@yahoo.com>.
\end{abstract}

\section{RÉSUMÉ}

Cet article a pour objectif le développement et la validation d'un instrument de mesure de la qualité. La revue de la littérature a permis de retenir 10 pratiques de management de la qualité ainsi que les items qui leur sont liés. L'étude empirique a touché 133 entreprises tunisiennes et a permis, par le biais d'une analyse factorielle confirmatoire, de retenir sept pratiques critiques de management de la qualité et de valider un instrument de mesure.

\begin{abstract}
The aim of this article is to develop and validate an instrument for measuring quality. From a review of the literature in the field of quality management, ten quality management practices were identified. An instrument measuring these practices was
\end{abstract}


developed. Data were obtained from a survey of 133 Tunisian companies. Based on confirmatory factor analysis, seven quality management practices were retained and the reliability and validity of the instrument were tested and validated.

\section{RESUMEN}

Este artículo tiene por objetivo el desarrollo y la validación de un instrumento de medida de la calidad. La revisión de la literatura nos permite obtener diez prácticas de la calidad de las empresas al igual que los ítems para medir esa calidad. El estudio empírico consta de 133 empresas tunecinas y permitió, a través de un análisis factorial confirmatorio, obtener siete prácticas críticas de gestión de la calidad y la validación de un instrumento de medida.

\section{ZUSAMMENFASSUNG}

Das Ziel dieses Artikels ist die Entwicklung und die Bewertung eines Instrumentes zur Qualitätsmessung. Anhand verschiedenster Literatur wurden zehn Praktiken des Qualitätsmanagements und die dazugehörigen Techniken festgehalten. Die empirische Studie wurde anhand von 133 tunesischen Unternehmen durchgeführt. Mit Hilfe einer Faktorenanalyse ist es gelungen sieben kritische Praktiken des Qualitätsmanagements zu bestimmen und ein geeignetes Messinstrument zu gestalten.

\section{Introduction}

Les mutations au plan de l'environnement ont poussé les entreprises à remettre en cause la façon traditionnelle de percevoir la qualité des produits et des services proposés aux clients et à se doter de mécanismes pour faire face à une concurrence de plus en plus féroce. Dans ce contexte, les spécialistes de la qualité ont proposé plusieurs démarches susceptibles d'améliorer la performance des entreprises. Ces démarches sont présentées sous forme d'un ensemble de pratiques de management de la qualité. Plusieurs auteurs, notamment Oberle (1990), Schaffer et Thomson (1992) et Kelly (1992) sont très critiques par rapport à ces démarches.

Une première critique concerne le fait que ces pratiques ne sont pas issues d'un cadre conceptuel rigoureux. Dans cet état d'esprit, plusieurs recherches tentent de démontrer l'impact des pratiques de management de la qualité sur la performance de l'entreprise. Malheureusement, ces études divergent fortement dans leurs conclusions. Une deuxième critique relève que les pratiques sont nombreuses et ne sont pas hiérarchisées. Il est donc difficile pour une entreprise qui introduit une démarche qualité de savoir comment procéder pour cheminer dans la voie de la qualité et notamment de savoir comment concentrer son énergie de façon prioritaire. Dans 
cet esprit, plusieurs auteurs ont relevé les pratiques «clés» ou «critiques» sur lesquelles repose le succès d'une démarche qualité (Saraph, Benson et Schroeder, 1989; Ahire, Golhar et Waller, 1996; Motwani et al., 1997; Forza et Filippini, 1998; Kannan et al., 1999; Zhang, Waszink et Wijngaard, 2000 ; Kaynak, 2003). Ces recherches n'apportent pas de réponses majoritaires quant aux pratiques les plus déterminantes susceptibles d'améliorer la performance de l'entreprise.

Par ailleurs, le diagnostic qualité réalisé auprès des entreprises tunisiennes par Lakhal et Limam (2000a) a permis de constater qu'il y a une volonté d'amélioration de la qualité, étant donné que ces entreprises sont conscientes et surtout convaincues de l'importance et de la pertinence de la qualité dans la réussite et la survie de l'entreprise. Toutefois, les démarches réalisées jusque-là par ces entreprises semblent peu compatibles avec une démarche qualité. Ce dysfonctionnement est dû à la méconnaissance des conditions à réunir pour l'instauration d'un management de la qualité (Lakhal et Limam, 2000a).

Pour aider les entreprises tunisiennes à emprunter la voie de la qualité, cette recherche a pour objectif:

- de relever les pratiques de management de la qualité,

- de développer sur la base de ces pratiques un instrument de mesure de la qualité,

- de valider empiriquement l'instrument de mesure de la qualité auprès des entreprises tunisiennes.

Quelques recherches similaires ont été menées dans le but de valider des instruments de mesure de la qualité à travers le monde, mentionnons entre autres Saraph, Benson et Schroeder (1989), Flynn, Schroeder et Sakakibara (1994), Ahire, Golhar et Waller (1996) et Zhang, Waszink et Wijngaard (2000). Ces instruments varient sur le plan des pratiques de management de la qualité et des items retenus. On propose, dans le cadre de cet article, de développer et de valider un instrument de mesure de la qualité propre aux entreprises tunisiennes du secteur de transformation des matières plastiques.

L'article est organisé de la manière suivante. Dans la première section, nous faisons une revue de la littérature portant sur les pratiques de management de la qualité. Dans la deuxième, nous décrivons la méthodologie de recherche. Dans la troisième section, nous rapportons les résultats obtenus. Enfin, dans la dernière section, nous présentons les conclusions, les limites et les voies futures de la recherche. 


\section{Les pratiques de management de la qualité : revue de la littérature}

L'étude des pratiques de management de la qualité a fait l'objet de nombreuses recherches. Les pratiques dégagées par les auteurs sont très nombreuses (Saraph, Benson et Schroeder, 1989; Flynn et al., 1994; Powell, 1995; Ahire, Golhar et Waller, 1996; Motwani et al., 1997; Dale et al.,1997; LaHay et Noble, 1998; Tamimi,1998; Forza et Filippini,1998; Choi et Eboch, 1998;Anderson et Sohal, 1999; Kannan et al., 1999;Zhang, Waszink et Wijngaard, 2000; Najmi et Kehoe, 2000; Sun, 2001; Kaynak, 2003). Toutefois, certaines pratiques sont de nature semblable. L'examen attentif des pratiques proposées permet de considérer 10 pratiques génériques distinctes, lesquelles apparaissent au tableau 1. Chacune de ces pratiques pouvant comprendre différentes facettes, elles seront opérationalisées à partir d'un certain nombre d'items.

\section{TABLEAU 1}

\section{Pratiques de management de la qualité}

\begin{tabular}{ll}
\hline Intitulé & Description \\
\hline $\begin{array}{l}\text { Engagement et soutien } \\
\text { de la haute direction }\end{array}$ & $\begin{array}{l}\text { Pratique reliée à l'engagement et au soutien de la } \\
\text { haute direction. }\end{array}$ \\
\hline Organisation pour la qualité & $\begin{array}{l}\text { Pratique reliée à la mise en place de la démarche } \\
\text { qualité. }\end{array}$ \\
\hline Formation des employés & Pratique reliée à la formation des employés. \\
\hline Participation des employés & Pratique reliée à la participation des employés. \\
\hline $\begin{array}{l}\text { Management de la qualité } \\
\text { des fournisseurs }\end{array}$ & $\begin{array}{l}\text { Pratique reliée au management de la qualité } \\
\text { des fournisseurs. }\end{array}$ \\
\hline Focalisation sur les clients & Pratique reliée à la focalisation sur les clients. \\
\hline Soutien continu & $\begin{array}{l}\text { Pratique visant à faire en sorte que la qualité soit } \\
\text { un processus continu, qu'elle ne se limite pas à un } \\
\text { projet ou à un programme avec un début et une fin. }\end{array}$ \\
\hline $\begin{array}{l}\text { Amélioration du système } \\
\text { qualité }\end{array}$ & $\begin{array}{l}\text { Pratique reliée à la gestion des améliorations } \\
\text { du système qualité. }\end{array}$ \\
\hline Information et analyse & $\begin{array}{l}\text { Pratique reliée au recueil, à la mesure et à l'analyse } \\
\text { des informations liées à la qualité. }\end{array}$ \\
\hline $\begin{array}{l}\text { Utilisation des techniques } \\
\text { statistiques de la qualité }\end{array}$ & $\begin{array}{l}\text { Pratique reliée à l'utilisation des techniques } \\
\text { statistiques de la qualité. }\end{array}$ \\
\hline
\end{tabular}


TABleau 2

Liens entre les pratiques retenues et celles proposées dans la littérature

\begin{tabular}{ll}
\hline Intitulé & Pratiques apparentées \\
\hline $\begin{array}{l}\text { Engagement } \begin{array}{l}\text { et soutien de la } \\
\text { haute direction }\end{array} \\
\text { Engagement de la haute direction (Ahire, Golhar et Waller, } \\
\text { Soh; Powell, 1995; Tamimi, 1998), leadership (Anderson et } \\
\text { pour la qualité }\end{array}$ & $\begin{array}{l}\text { Design du management de la qualité (Ahire, Golhar et } \\
\text { Waller, 1996), organisation ouverte (Powell, 1995), groupes } \\
\text { transfonctionnels (LaHay et Noble, 1998), contrôle et amélio- } \\
\text { ration des processus (Zhang, Waszink et Wijngaard, 2000). }\end{array}$ \\
\hline $\begin{array}{l}\text { Formation } \\
\text { des employés }\end{array}$ & $\begin{array}{l}\text { Formation (Saraph, Benson et Schroeder, 1989), éducation } \\
\text { et/ou formation (Ahire, Golhar et Waller, 1996; Kannan et al., }\end{array}$ \\
& $\begin{array}{l}\text { 1999; Powell, 1995; Tamimi, 1998; Zhang, Waszink } \\
\text { et Wijngaard, 2000). }\end{array}$ \\
\hline $\begin{array}{l}\text { Participation } \\
\text { des employés }\end{array}$ & $\begin{array}{l}\text { Participation (Zhang, Waszink et Wijngaard, 2000), déléga- } \\
\text { tion (Ahire, Golhar et Waller, 1996; Powell, 1995), implica- } \\
\text { tion des employés (Ahire, Golhar et Waller, 1996), relations } \\
\text { avec les employés (Saraph, Benson et Schroeder, 1989). }\end{array}$
\end{tabular}

Management Management de la qualité des fournisseurs (Ahire, Golhar de la qualité et Waller, 1996; Saraph, Benson et Schroeder, 1989; Zhang, des fournisseurs Waszink et Wijngaard, 2000), management des fournisseurs (Tamimi, 1998), fournisseurs (Najmi et Kehoe, 2000; Sun, 2001), relations (liens) avec les fournisseurs (Forza et Filippini, 1998; Powell 1995).

Focalisation $\quad$ Focalisation sur les clients (Ahire, Golhar et Waller, 1996; sur les clients Anderson et Sohal, 1999; LaHay et Noble, 1998; Zhang, Waszink et Wijngaard, 2000), relation forte avec les clients (Powell, 1995), satisfaction des clients (Forza et Filippini, 1998).

Soutien continu Amélioration continue (Douglas and Judge, 2001), reconnaissances et récompenses (Zhang, Waszink et Wijngaard, 2000).

Amélioration Amélioration du système qualité (Zhang, Waszink et du système qualité Wijngaard, 2000).

Information Information et analyse (Anderson et Sohal, 1999; Choi et et analyse Eboch, 1998), information (Sun, 2001), flux d'information (Kannan et al., 1999), système d'information sur la qualité (Najmi et Kehoe, 2000), mesure des processus (LaHay et Noble, 1998), utilisation des informations internes sur la qualité (Ahire, Golhar et Waller, 1996), données sur la qualité (Saraph, Benson et Schroeder, 1989), mesure de la qualité (Powell, 1995), benchmarking (Ahire, Golhar et Waller, 1996; Powell, 1995).

Utilisation des Utilisation des outils et techniques de la qualité

techniques statisti- (Ahire, Golhar et Waller, 1996), méthodes de la qualité ques de la qualité totale (Douglas et Judge, 2001). 
Le tableau 2 établit des liens entre les pratiques considérées dans ce travail et les pratiques proposées dans d'autres recherches. De façon plus précise, nous mentionnons pour chaque pratique considérée la liste de pratiques similaires (ou apparentées) proposées par d'autres auteurs. Cette liste sert à illustrer les bases de nos pratiques génériques. Cette liste a également fortement inspiré la définition des items servant à opérationaliser chaque pratique (voir annexe).

\subsection{Engagement et soutien de la haute direction}

L'engagement de la direction générale est le préalable le plus important pour l'instauration d'une approche qualité, en ce sens que le non-consentement de la direction générale à tout projet proposé veut dire l'échec total de ce dernier. En effet, Ahire et O'Shaughnessy (1998) précisent que la haute direction joue le rôle de pivot dans l'accroissement du succès du management de la qualité.

Leonard et Sasser (1983) soulignent que la direction générale doit être directement et activement impliquée dans l'amélioration de la qualité. Feigenbaum (1984) considère l'engagement de la haute direction comme étant le moyen idéal pour promouvoir l'engagement organisationnel pour l'amélioration de la qualité. Crosby (1986) croit que personne ne peut réussir dans une approche qualité sans l'ombre portée de la haute direction. Bertram (1991) témoigne que le manque d'engagement des cadres supérieurs est la raison majeure de l'échec de $80 \%$ des tentatives d'implantation du management de la qualité.

\subsection{Organisation pour la qualité}

Kelada (1996) soutient que la mise en place de la qualité totale engendre les transformations suivantes: la création d'un comité de direction pour gérer la qualité totale et la nomination d'un coordinateur ou d'un comité de coordination. Ces transformations entraînent des changements au niveau de la structure organisationnelle.

Gonthier (1992) stipule que la mise en place d'un management par la qualité totale chez Hydro-Québec a permis la création de 65 équipes d'amélioration de la qualité, dont les membres appartiennent à une même unité administrative, ainsi que de trois équipes de projet nommées en raison de leur compétence ou de leur expérience. Wruck et Jensen (1997) qualifient le management de la qualité de révolution organisationnelle. 
Glover (1993) considère le comité qualité comme étant une formule efficace pour planifier et mettre en place un management de la qualité. La composition du comité est très importante et doit inclure des personnes comme les leaders des groupes qualité, les opérationnels, etc. Le comité doit communiquer les problèmes et les décisions de façon quotidienne durant l'implantation.

Les recherches réalisées sur le thème de la qualité établissent un lien entre la mise en place d'une approche qualité et le changement dans la structure organisationnelle. C'est ainsi que Lakhal et Limam (2000b) montrent, dans le cas des entreprises tunisiennes, que la mise en place d'une stratégie qualité s'est accompagnée de transformations au niveau de la structure organisationnelle.

\subsection{Formation des employés}

L'importance de la formation est relevée chez les principaux spécialistes de la qualité; ainsi, la formation permanente des cadres et de tous les membres de l'organisation figure parmi les 14 points de Deming et le lancement d'un programme massif de formation ressort dans l'approche de management de la qualité de Juran.

Ishikawa (1984) insiste sur le fait que la maîtrise de la qualité commence par l'éducation et finit par l'éducation. Gogue (1990) note que la formation permet de donner aux travailleurs la satisfaction du travail bien fait, le plaisir de coopérer avec leurs semblables et la joie de progresser.

Montaigne et Stora (1986) précisent que si l'on veut que la formation soit efficace au sein de l'organisation, il faut qu'elle soit conçue de façon cohérente au niveau de l'entreprise et qu'elle soit adaptée à tous les membres de l'organisation pour qu'ils reçoivent sans exception une formation qualité correspondant à leurs besoins.

Thiagarajan et Zairi (1997) affirment que la formation permet de préparer les employés à une plus grande implication aux questions rattachées à la qualité. C'est pourquoi il devient crucial pour l'entreprise de fournir une formation qualité à ses employés. Cela donne un aperçu de l'importance d'un tel préalable, dans la mesure où la formation permet l'acquisition de connaissances et le développement d'habiletés nouvelles. Elle permet de modifier les façons de faire, les comportements et la culture. 


\subsection{Participation des employés}

Pour réussir dans un contexte de plus en plus turbulent, les managers doivent impérativement tirer profit de toutes les potentialités et compétences existantes au sein de l'entreprise. Pour ce faire, il devient primordial pour l'organisation d'adopter le management participatif.

Kelada (1992) est d'avis que la gestion participative repose sur un certain nombre de principes, dont les suivants:

- La participation engendre la motivation.

- Le travail d'équipe est indispensable à une gestion efficace.

- Le fonctionnement par équipe requiert une coordination adéquate.

- La gestion participative est basée sur une collaboration de la direction générale, avec l'ensemble des employés, à tous les niveaux hiérarchiques.

- Les relations entre la direction et le personnel sont basées sur la confiance et non sur l'autorité.

- La direction encourage la circulation libre de l'information.

- Les conflits sont examinés en termes de problèmes à résoudre.

- La direction par domination est remplacée par l'autocontrôle.

- L'individu est responsable d'objectifs spécifiques, reflétant les objectifs de l'entreprise.

\subsection{Management de la qualité des fournisseurs}

Deming (1991), Juran (1983) et Ishikawa (1984) insistent sur la création de partenariat avec les fournisseurs. Les spécialistes de la qualité stipulent que l'entreprise doit choisir ses fournisseurs sur la base de la qualité et suggèrent que l'entreprise travaille directement avec les fournisseurs pour s'assurer de la qualité des matières fournies.

Dyer et Ouchi (1993) soutiennent que la réussite des entreprises japonaises est en grande partie liée à la structure relationnelle dont elles disposent avec leurs fournisseurs. Ces entreprises aident leurs fournisseurs à atteindre une qualité acceptable des produits et services. 


\subsection{Focalisation sur les clients}

L'importance de l'orientation clients apparaît dans la définition proposée généralement au concept de la qualité et aussi dans l'interaction des trois coins du triangle de Deming (1991), à savoir:1) le produit, 2) la disposition de formation pour les clients et 3 ) le client et la manière avec laquelle le produit est utilisé. En outre, Neuville (1996) signale que le client devient ipso facto le nouveau maître dont il faut satisfaire toutes les exigences.

\subsection{Soutien continu}

Le management de la qualité suppose un effort prodigieux de la part de tous dans l'entreprise et à tous les niveaux hiérarchiques. Tischler (1996) suggère que les employés et la direction générale doivent être guidés par le challenge pour faire toujours mieux les choses, afin de satisfaire les clients. Nwabueze et Kanji (1997) mentionnent que certaines entreprises nomment des coordinateurs afin de soutenir les efforts qualité, dans le but de mieux véhiculer les exigences du management de la qualité et les attentes de la direction générale à tous les niveaux hiérarchiques.

Nwabueze (1996) souligne l'importance de développer une stratégie qualité, ayant pour but de faire de la qualité une partie intégrante de chaque individu et de chaque groupe et d'encourager tous les membres de l'entreprise à créer une culture basée sur la satisfaction des besoins et désirs des clients.

\subsection{Amélioration du système qualité}

Le système qualité est défini comme étant la structure, les procédures, les processus et les ressources nécessaires à l'implantation d'un management de la qualité. En effet, toutes les entreprises s'appuient sur une structure de management existante, qui devrait former la base sur laquelle est édifié le système qualité.

Zhang, Waszink et Wijngaard (2000) notent que l'importance de cette pratique se fait surtout sentir lorsque l'entreprise opère dans un cadre orienté vers la certification ISO 9000. C'est le cas des entreprises tunisiennes dans la mesure où l'on compte actuellement près de 400 entreprises certifiées.

\subsection{Information et analyse}

Cette pratique a été distinguée dans plusieurs études relatives à l'identification des pratiques de management de la qualité, dont celles de Schroeder 
et Sakakibara (1994) et de Powell (1995). De nos jours, plusieurs organisations utilisent l'analyse comparative ou benchmarking comme un outil pour obtenir des informations concernant l'amélioration des processus internes et les concurrents. Weill (2001) définit le benchmarking comme étant un processus continu et systématique d'évaluation des produits, des services et des méthodes par rapport à ceux des concurrents les plus sérieux et des entreprises reconnues comme leaders ou chef de file. Thiagarajan et Zairi (1997) relèvent l'existence de quatre types essentiels de benchmarking, à savoir:

- le benchmarking compétitif: comparaison avec les meilleurs concurrents.

- le benchmarking fonctionnel: comparaison avec les mêmes fonctions ou processus chez les leaders dans la même industrie.

- le benchmarking générique : comparaison avec les mêmes fonctions ou processus sachant le type d'industrie.

- le benchmarking interne.

\subsection{Utilisation des techniques statistiques de la qualité}

Les spécialistes de la qualité, tels que Deming (1991), Juran (1983) et Ishikawa (1984), soulignent la nécessité pour l'entreprise d'utiliser des outils statistiques pour analyser les processus de travail. Divers outils statistiques peuvent être utilisés pour l'amélioration de la qualité : les cartes de contrôle, la courbe de Pareto, le diagramme des causes à effets, etc.

Deming (1991) précise que l'utilisation des techniques statistiques aide les entreprises orientées vers la qualité à contrôler les variations et à dégager les zones critiques d'amélioration.

\section{Méthodologie de recherche}

L'objectif de cet article est de développer et de valider, à travers une étude empirique, un instrument de mesure de la qualité propre aux entreprises tunisiennes. Pour ce faire, un questionnaire composé de 43 items répartis sur 10 pratiques de management de la qualité a été élaboré. L'étude s'est focalisée sur la totalité des entreprises tunisiennes qui font de la transformation des matières plastiques et qui sont au nombre de 133. Le choix de ce secteur est dû au fait que plusieurs entreprises ont mis en place des approches d'amélioration de la qualité. Ces entreprises sont caractérisées par une taille réduite. Ainsi, $50 \%$ des entreprises ont moins de 20 salariés et près de $80 \%$ des entreprises, moins de 50 salariés. 
Par ailleurs, un prétest a été réalisé auprès de 10 entreprises afin de valider le questionnaire ainsi développé, ce qui a permis de réviser et de vérifier l'importance et la pertinence de certaines questions. La collecte des données a été effectuée en utilisant la méthode du contact direct avec le directeur général des entreprises étudiées. En effet, les managers des entreprises enquêtées ont été contactés par téléphone pour fixer un rendez-vous; les managers ont manifesté un engouement sans précédent pour la recherche réalisée. L'analyse des données a été réalisée avec les logiciels SPSS 10.0 et Lisrel 8.30 pour Windows, afin de purifier les items et de proposer un instrument fiable et valide de mesure de la qualité.

\section{Résultats et discussions}

\subsection{Identification des pratiques de management de la qualité}

L'identification des pratiques de management de la qualité a été réalisée en suivant les deux analyses statistiques distinctes suivantes:

- l'analyse factorielle en composantes principales afin de faire émerger une structure factorielle;

- l'analyse factorielle confirmatoire afin de confirmer la structure factorielle.

Le tableau 3 présente les résultats des analyses susmentionnées. L'analyse factorielle en composantes principales réalisée grâce au logiciel SPSS montre que plusieurs items peuvent être éliminés en raison de la faiblesse de leur valeur propre. Grandzol et Gershon (1998) précisent que les items avec valeurs propres multiples très élevées doivent être traités de la même manière que les items qui disposent de valeurs propres faibles.

Pour valider la structure factorielle qui a émergé de l'analyse factorielle en composantes principales, on a réalisé une analyse factorielle confirmatoire moyennant le logiciel Lisrel. Les contributions factorielles sont présentées au tableau 3. Papke-Shields et Malhotra (2001) signalent que les items qui disposent d'une contribution factorielle inférieure à 0,6 doivent être éliminés de l'analyse.

L'analyse factorielle confirmatoire confirme les résultats de l'analyse factorielle en composantes principales. En effet, les résultats montrent que certains items n'atteignent pas le seuil de 0,6 (relevés en gras) et doivent, par conséquent, être éliminés de l'analyse ; il s'agit des items suivants : STM5, OPQ2, OPQ3, OPQ4, FDE4, MQF1, MQF2, MQF3, MQF4, SC1, SC2, SC3, SC4, ASQ1, UTSQ1, UTSQ2, UTSQ3, UTSQ4, UTSQ5. 
Par ailleurs, l'analyse révèle que trois pratiques de management de la qualité seront éliminées : management de la qualité des fournisseurs, soutien continu et utilisation des techniques statistiques de la qualité. Compte tenu de cette analyse en deux étapes, l'instrument final de mesure de la qualité sera composé de sept pratiques de management de la qualité, à savoir: 1) engagement et soutien de la haute direction,2) organisation pour la qualité, 3) formation des employés, 4) participation des employés, 5) focalisation sur les clients, 6) amélioration du système qualité, 7) information et analyse de 24 items.

\section{TABLEAU 3}

Sélection des pratiques de management de la qualité

\begin{tabular}{|c|c|c|c|c|}
\hline Pratiques qualité & Items & Facteur 1 & Facteur 2 & Contribution \\
\hline \multirow{5}{*}{$\begin{array}{l}\text { Engagement et soutien } \\
\text { de la haute direction (STM) }\end{array}$} & STM1 & 0,779 & 0,096 & 0,75 \\
\hline & \multirow{4}{*}{$\begin{array}{l}\text { STM2 } \\
\text { STM3 } \\
\text { STM4 } \\
\text { STM5 }\end{array}$} & 0,681 & 0,195 & 0,63 \\
\hline & & 0,811 & 0,08 & 0,85 \\
\hline & & 0,744 & 0,184 & 0,70 \\
\hline & & 0,006 & 0,972 & 0,07 \\
\hline \multirow[t]{5}{*}{ Organisation pour la qualité (OPQ) } & \multirow{5}{*}{$\begin{array}{l}\text { OPQ1 } \\
\text { OPQ2 } \\
\text { OPQ3 } \\
\text { OPQ4 } \\
\text { OPQ5 }\end{array}$} & 0,903 & 0,201 & 0,88 \\
\hline & & 0,221 & 0,861 & 0,44 \\
\hline & & 0,186 & 0,707 & 0,44 \\
\hline & & 0,003 & 0,853 & 0,14 \\
\hline & & 0,902 & 0,196 & 0,90 \\
\hline \multirow[t]{5}{*}{ Formation des employés (FDE) } & \multirow{5}{*}{$\begin{array}{l}\text { FDE1 } \\
\text { FDE2 } \\
\text { FDE3 } \\
\text { FDE4 } \\
\text { FDE5 }\end{array}$} & 0,711 & & 0,70 \\
\hline & & 0,742 & & 0,82 \\
\hline & & 0,736 & & 0,64 \\
\hline & & 0,343 & & 0,38 \\
\hline & & 0,654 & & 0,61 \\
\hline \multirow[t]{3}{*}{ Participation des employés (PDE) } & \multirow{3}{*}{$\begin{array}{l}\text { PDE1 } \\
\text { PDE2 } \\
\text { PDE3 }\end{array}$} & 0,822 & & 0,68 \\
\hline & & 0,821 & & 0,71 \\
\hline & & 0,868 & & 0,91 \\
\hline \multirow{4}{*}{$\begin{array}{l}\text { Management de la qualité } \\
\text { des fournisseurs (MQF) }\end{array}$} & MQF1 & 0,019 & 0,893 & 0,17 \\
\hline & \multirow{3}{*}{$\begin{array}{l}\text { MQF2 } \\
\text { MQF3 } \\
\text { MQF4 }\end{array}$} & 0,605 & 0,659 & 0,39 \\
\hline & & 0,848 & 0,779 & 0,45 \\
\hline & & 0,801 & 0,608 & 0,36 \\
\hline \multirow[t]{4}{*}{ Focalisation sur les clients (FSC) } & \multirow{4}{*}{$\begin{array}{l}\text { FSC1 } \\
\text { FSC2 } \\
\text { FSC3 } \\
\text { FSC4 }\end{array}$} & 0,773 & & 0,78 \\
\hline & & 0,818 & & 0,82 \\
\hline & & 0,801 & & 0,68 \\
\hline & & 0,709 & & 0,61 \\
\hline
\end{tabular}




\begin{tabular}{lcccc}
\hline Pratiques qualité & Items & Facteur 1 & Facteur 2 & Contribution \\
\hline Soutien continu (SC) & SC1 & 0,358 & 0,735 & 0,32 \\
\cline { 3 - 5 } & SC2 & 0,657 & 0,745 & 0,37 \\
\cline { 3 - 5 } & SC3 & 0,802 & 0,652 & 0,36 \\
\cline { 3 - 5 } & SC4 & 0,164 & 0,897 & 0,43 \\
\hline Amélioration du système & ASQ1 & 0,109 & & 0,42 \\
\cline { 3 - 5 } qualité (ASQ) & ASQ2 & 0,799 & & 0,76 \\
\cline { 3 - 5 } & ASQ3 & 0,793 & & 0,75 \\
\cline { 3 - 5 } & ASQ4 & 0,778 & & 0,75 \\
\cline { 3 - 5 } & IEA1 & 0,735 & & 0,66 \\
\cline { 3 - 5 } & IEA2 & 0,751 & & 0,66 \\
\cline { 3 - 5 } & IEA3 & 0,778 & & 0,73 \\
\cline { 3 - 5 } & IEA4 & 0,855 & & 0,88 \\
\cline { 3 - 5 } & UTSQ1 & 0,099 & 0,996 & 0,12 \\
\cline { 3 - 5 } & UTSQ2 & 0,378 & 0,716 & 0,23 \\
\cline { 3 - 5 } statistiques de la qualité (UTSQ) et analyse (IEA) & UTSQ3 & 0,244 & 0,836 & 0,39 \\
\cline { 3 - 5 } & UTSQ4 & 0,745 & 0,673 & 0,42 \\
\cline { 3 - 5 } & UTSQ5 & 0,853 & 0,703 & 0,41 \\
\hline
\end{tabular}

\subsection{Validité de l'instrument de mesure de la qualité}

On propose dans ce qui suit de tester empiriquement la validité de l'instrument de mesure sur la base des sept pratiques de management de la qualité restantes. Pour ce faire, les étapes suggérées par O'Learly-Kelly et Vokurka (1998) ont été poursuivies. C'est ainsi qu'on procède au test de l'unidimensionalité, de la fiabilité et de la validité convergente et discriminante des variables latentes (pratiques de management de la qualité) qui composent notre instrument de mesure.

Avant de procéder à l'analyse de la validité de construit, on réalisera au préalable un test de multinormalité. Les résultats sont présentés au tableau 4.

TABleau 4

Test de normalité

\begin{tabular}{lccccccc}
\hline & IEA & ASQ & OPQ & FDE & PDE & FSC & STM \\
\hline $\mathbf{N}$ & 92 & 92 & 92 & 92 & 92 & 92 & 92 \\
Moyenne & 3,42 & 3,37 & 4,26 & 3,59 & 3,84 & 3,81 & 4,05 \\
Écart type & 0,94 & 1,17 & 0,85 & 0,90 & 0,92 & 0,82 & 0,79 \\
Skewness & $-0,58$ & $-0,25$ & $-1,09$ & $-0,66$ & $-1,14$ & $-1,00$ & $-0,56$ \\
Kurtosis & 0,11 & $-1,03$ & 0,59 & 0,64 & 1,13 & 0,85 & 0,03 \\
\hline
\end{tabular}


Kline (1998) précise que si les indices de Skewness et Kurtosis se trouvent dans la limite acceptable de $[-3 ;+3]$, la normalité est vérifiée. Les résultats du tableau 4 permettent de confirmer que toutes les variables suivent une distribution normale.

On propose maintenant de tester l'unidimensionalité, la fiabilité et la validité des échelles de mesure qui composent notre instrument de mesure de la qualité.

\subsubsection{Analyse de l'unidimensionalité}

Pour tester l'unidimensionalité, une analyse en composantes principales a été réalisée pour chaque variable latente retenue. Les items retenus, après purification, pour chaque pratique de management de la qualité permettent d'extraire un seul facteur. Ces résultats confirment l'unidimensionalité des échelles de mesure qui composent notre instrument de mesure des pratiques de management de la qualité.

\subsubsection{Analyse de la fiabilité}

Ayant mentionné les coefficients alpha de Cronbach pour chaque pratique de management de la qualité, on propose dans le cadre de cette deuxième étude empirique de calculer aussi le rhô de cohérence interne de Jöreskog, dans la mesure où il est plus adapté à l'analyse selon la méthode des équations structurelles. Les résultats apparaissent au tableau 5.

\section{TABLEAU 5}

\section{Coefficients de fiabilité}

\begin{tabular}{lc}
\hline Variables latentes & Rhô de Jöreskog \\
\hline Engagement et soutien de la haute direction & 0,81 \\
Organisation pour la qualité & 0,90 \\
Formation des employés & 0,78 \\
Participation des employés & 0,81 \\
Focalisation sur les clients & 0,83 \\
Information et analyse & 0,82 \\
Amélioration du système qualité & 0,80 \\
\hline
\end{tabular}

Les résultats indiquent, pour toutes les échelles de notre instrument de mesure, que le coefficient rhô de cohérence interne présente des valeurs supérieures à 0,8 , à l'exception de l'échelle «formation des employés » qui en est toutefois très près. Ce qui confirme la fiabilité de toutes les pratiques de management de la qualité retenues. 


\subsubsection{Analyse de la validité convergente}

La validité convergente a été estimée en utilisant l'approche de Fornell et Larcker (1981). En effet, les résultats du tableau 3 montrent que toutes les contributions factorielles sont statistiquement significatives au seuil $\alpha$ de $1 \%$ (p-valeur $<0,01)$.

Ensuite, le rhô de validité convergente a été calculé pour chaque pratique de management de la qualité. Les résultats sont présentés au tableau 6.

TABLeau 6

Validité convergente

\begin{tabular}{lc}
\hline Variables latentes & $\begin{array}{c}\text { Pourcentage de } \\
\text { variance moyenne extraite }\end{array}$ \\
\hline Engagement et soutien de la haute direction & 0,53 \\
Organisation pour la qualité & 0,81 \\
Formation des employés & 0,49 \\
Participation des employés & 0,60 \\
Focalisation sur les clients & 0,56 \\
Information et analyse & 0,54 \\
Amélioration du système qualité & 0,57 \\
\hline
\end{tabular}

La validité convergente est bonne et la variance extraite de chaque échelle ou variable latente est acceptable. Les résultats du tableau 6 révèlent que la variance extraite dépasse le seuil de 0,5 pour toutes les échelles de mesure, à l'exception de celle de l'échelle formation des employés pour qui le pourcentage de la variance extraite est de $49 \%$.

\subsubsection{Analyse de la validité discriminante}

Ferrandi et Valette-Florence (2002) sont d'avis qu'un construit a une validité discriminante satisfaisante lorsque le modèle testé, en laissant libre les corrélations entre les variables latentes, a de meilleurs indices d'ajustement qu'un modèle où l'on fixerait à 1 les corrélations entre ces variables. Le tableau 7 présente les résultats du test de la validité discriminante.

\section{TABLEAU 7}

\section{Validité discriminante}

\begin{tabular}{lcc}
\hline Modèles testés & Chi carré & Degrés de liberté (ddl) \\
\hline Modèle 1 (corrélations libres) & 410,15 & 231 \\
\hline Modèle 2 (corrélations fixées à 1) & 963,85 & 252 \\
\hline Différence (modèle 2 - modèle 1) & 553,7 & 21 \\
\hline
\end{tabular}


La différence de chi carré est statistiquement significative au seuil alpha de $1 \%$. Ce test confirme la validité discriminante des variables latentes qui composent notre instrument de mesure des pratiques de management de la qualité et montre que le modèle avec corrélations libres dispose d'un meilleur ajustement des données par rapport au modèle avec corrélations fixées à 1 .

En conclusion, notre outil de mesure épuré grâce à l'analyse factorielle exploratoire en composantes principales est considéré valide selon l'analyse factorielle confirmatoire. Notre outil final de mesure comprend 24 items regroupés dans les 7 pratiques suivantes: 1) engagement et soutien de la haute direction, 2) organisation pour la qualité, 3) formation des employés, 4) participation des employés, 5) focalisation sur les clients, 6) amélioration du système qualité, 7) information et analyse.

\subsection{Discussions}

L'instrument de mesure de la qualité ainsi développé et validé se révèle différent des instruments précédemment mis au point par Saraph, Benson et Schroeder (1989); Flynn, Schroeder et Sakakibara (1994); Ahire, Golhar et Waller (1996); Zhang, Waszink et Wijngaard (2000). Le tableau 8 présente une comparaison entre ces instruments sur le plan des pratiques retenues. En effet, pour la validité interne, cet instrument est meilleur que les instruments de Saraph, Benson et Schroeder (1989), Flynn, Schroeder et Sakakibara (1994) et de Zhang, Waszink et Wijngaard (2000). Tous ces auteurs ont essayé de développer des instruments de mesure de la qualité à partir de l'étude d'entreprises appartenant à différents secteurs d'activité. Ces instruments disposent par conséquent d'une meilleure validité externe.

L'instrument de mesure de la qualité développé par Ahire, Golhar et Waller (1996) est meilleur que l'instrument développé dans le cadre de cette recherche, pour la validité interne, dans la mesure où ces auteurs ont utilisé des données recueillies auprès de 371 entreprises industrielles, alors que cet instrument a été développé et validé à partir de 133 entreprises industrielles tunisiennes. Toutefois, notre instrument comporte deux caractéristiques majeures: premièrement, il tient compte des pratiques de management de la qualité retenues dans les quatre instruments, afin de suivre les recommandations de Ahire, Golhar et Waller (1996); deuxièmement, il est meilleur pour les entreprises tunisiennes de transformation des matières plastiques. 


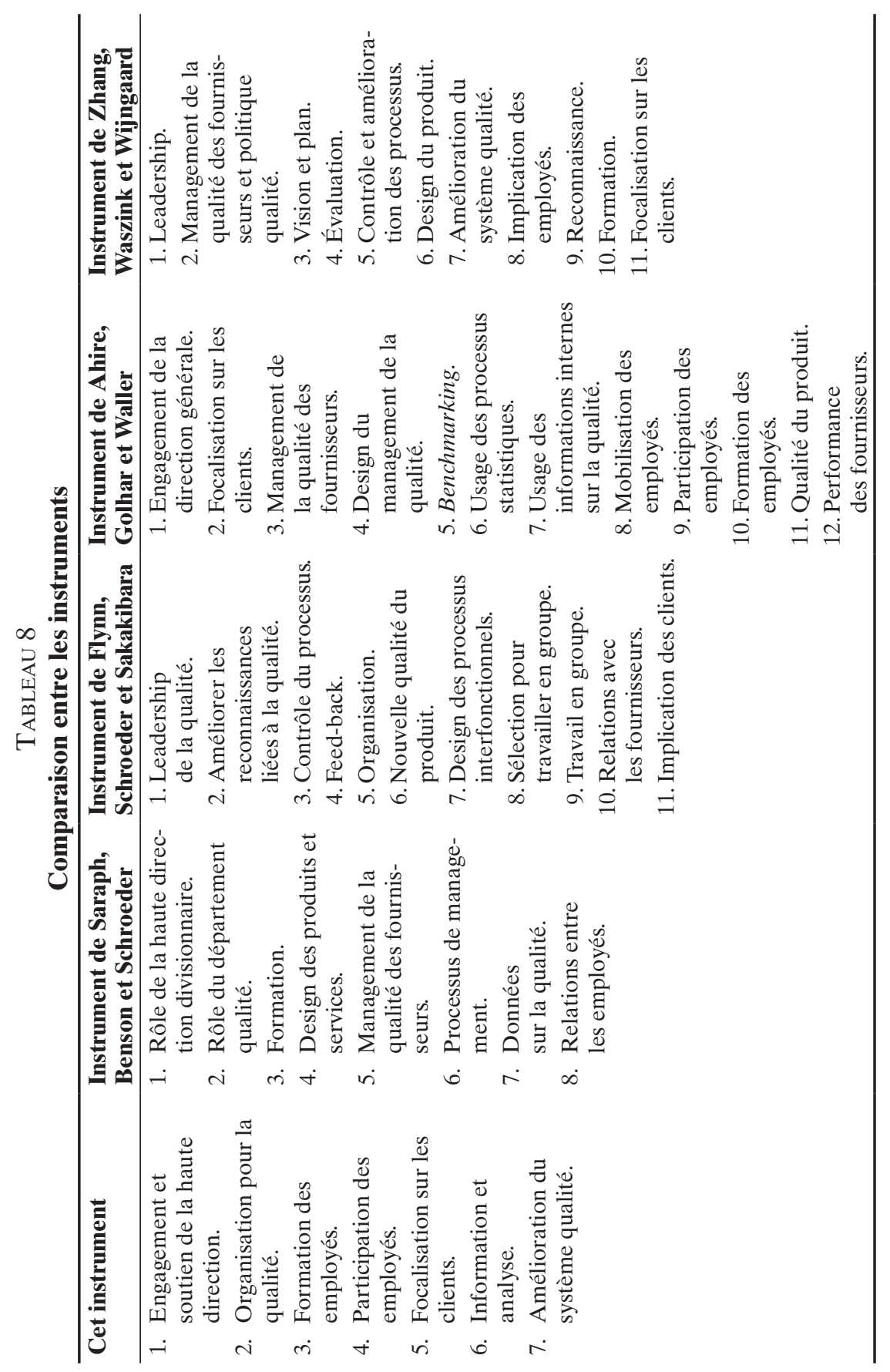

Revue internationale P.M.E., vol. 20, nº 2, 2007 


\section{Conclusions, limites et voies futures de la recherche}

Cette recherche a pour objectif le développement et la validation d'un instrument de mesure de la qualité dans les entreprises tunisiennes. Pour ce faire, une large revue de la littérature a été menée dans le but d'étudier et de sélectionner les pratiques de management de la qualité qui feront partie de l'instrument de mesure. L'étude théorique a touché les pratiques de management de la qualité utilisées dans les prix de la qualité les plus connus, ainsi que les pratiques de management de la qualité dégagées à la suite de nombreuses recherches empiriques dans plusieurs pays.

L'instrument théorique de mesure de la qualité ainsi développé a ensuite été testé et validé auprès de 133 entreprises tunisiennes de transformation des matières plastiques. En effet, on a commencé par une étude empirique afin d'épurer les items des pratiques de management de la qualité sélectionnées en réalisant une analyse factorielle exploratoire en composantes principales et une analyse factorielle confirmatoire. Les résultats de ces analyses ont permis d'éliminer 19 items et 3 pratiques de management de la qualité. L'instrument final de mesure est composé des pratiques suivantes: engagement et soutien de la haute direction, organisation pour la qualité, formation des employés, participation des employés, focalisation sur les clients, amélioration du système qualité, information et analyse, ainsi que de 24 items.

L'instrument de mesure de la qualité ainsi développé est utile aussi bien pour les praticiens que pour les chercheurs. Les managers des entreprises peuvent l'utiliser pour évaluer leur programme d'implantation de la qualité, déterminer le degré de maturité du management de la qualité au sein de leur entreprise et identifier les zones qui requièrent une attention particulière.

La limite de cet article réside dans le fait que cette étude a été réalisée sur un seul secteur d'activité, ce qui n'est pas représentatif et pose le problème de généralisation des résultats. Cet article permet d'ouvrir des pistes de recherches et d'autres études plus approfondies pourraient être envisagées afin d'élaborer d'autres instruments de mesure de la qualité dans les entreprises tunisiennes. Pour améliorer la validité externe de cet instrument, les recherches futures peuvent le tester dans différents secteurs d'activité. Ce genre de recherche permettrait d'apporter beaucoup d'informations pertinentes sur la manière dont chaque branche d'activité perçoit le management de la qualité. 
AnNEXe

\section{Engagement et soutien de la haute direction}

STM1. La direction générale est activement impliquée dans l'amélioration de la qualité (Crosby, 1986; Saraph, Benson et Schroeder, 1989; Glover, 1993;Anderson, Rungtusanatham et Schroeder, 1994;Badri, Davis et Davis, 1995; Thiagarajan et Zairi, 1997; Deleryd, Deltin et Klefsjo, 1999; Dean et Yunus, 1999; Rahman, 2001).

STM2. Le management fournit les ressources nécessaires à l'accomplissement adéquat des activités (Anderson, Rungtusanatham et Schroeder, 1994; Ahire, Golhar et Waller, 1996; Tan et al., 1999; Zhang, Waszink et Wijngaard, 2000; Kaynak, 2003).

STM3. La direction générale incite les employés pour la prise en compte des besoins et attentes des clients (Legendre et Beaucage, 1997; Dean et Yunus, 1999).

STM4. Les objectifs qualité de la direction sont diffusés à tous les employés (Mann et Kehoe, 1994; Handfield, Ghosh et Fawcett, 1998; Kannan et al., 1999; Tan et al., 1999).

STM5. La haute direction poursuit des objectifs à long terme (Saraph, Benson et Schroeder, 1989; Badri, Davis et Davis, 1995; Zhang, Waszink et Wijngaard, 2000).

\section{Organisation pour la qualité}

OPQ1. L'organisation dispose d'une méthode de gestion des processus (Juran, 1983; Ishikawa, 1984; Deming, 1991; Olian et Rynes, 1991; Glover, 1993; Dean et Bowen, 1994; Hackman et Wageman, 1995; Legendre et Beaucage, 1997; Easton et Jarrell, 1998; Bowman, 1998).

OPQ2. Tous les processus sont améliorés de façon continue (Dean et Bowen, 1994; Anderson, Rungtusanatham et Schroeder, 1994; Gummer, 1996; Legendre et Beaucage, 1997; Bowman, 1998).

OPQ3. Il y a une utilisation fréquente des groupes transdépartementaux (Juran, 1983; Ishikawa, 1984; Deming, 1991; Olian et Rynes, 1991; Powell, 1995; Ahire, Golhar et Waller, 1996; Easton et Jarrell, 1998; Kannan et al., 1999; Zhang, Waszink et Wijngaard, 2000). 
OPQ4. L'organisation utilise les cercles de qualité (Ishikawa, 1984; Saraph, Benson et Schroeder, 1989; Badri, Davis et Davis, 1995; Ahire, Golhar et Waller, 1996; Dean et Yunus, 1999; Zhang, Waszink et Wijngaard, 2000).

OPQ5. Il y a peu de bureaucratie (hiérarchie formelle, procédures et règles détaillées) au niveau de l'organisation (Powell, 1995; Tata et Prasad, 1998).

\section{Formation des employés}

FDE1. L'entreprise assure une formation continue pour son personnel d'encadrement (Saraph, Benson et Schroeder, 1989; Blackburn et Rosen, 1993; Mann et Kehoe, 1994; Badri, Davis et Davis, 1995; Powell, 1995; Wruck et Jensen, 1997; Easton et Jarrell, 1998; Rahman, 2001).

FDE2. L'entreprise assure une formation continue pour son personnel d'exécution (Juran, 1983; Ishikawa, 1984; Crosby, 1986; Saraph, Benson et Schroeder, 1989; Deming, 1991; Olian et Rynes, 1991; Blackburn et Rosen, 1993; Wruck et Jensen, 1997; Easton et Jarrell, 1998).

FDE3. Les besoins en formation sont toujours évalués (Legendre et Beaucage, 1997).

FDE4. Les employés bénéficient de congés de formation.

FDE5. L'entreprise mesure la satisfaction des employés au sujet des formations reçues (Legendre et Beaucage, 1997).

\section{Participation des employés}

PDE1. Les employés sont encouragés pour être totalement impliqués (Kelada, 1992; Blackburn et Rosen, 1993; Dean et Bowen, 1994; Davis et Fisher, 1994; Ahire, Golhar et Waller, 1996; Kelada, 1996; Wruck et Jensen, 1997; Zhang, Waszink et Wijngaard, 2000; Nilsson, Johnson et Gustafsson, 2001).

PDE2. Le management permet aux employés de participer dans l'achèvement des objectifs organisationnels (Olian et Rynes, 1991; Kelada, 1992; Glover, 1993 ; Blackburn et Rosen, 1993 ; Davis et Fisher, 1994 ; Hackman et Wageman, 1995; Kelada, 1996; Rahman, 2001;Nilsson, Johnson et Gustafsson, 2001). 
PDE3. Les employés sont les responsables des tâches qu'ils réalisent et sont les inspecteurs de leurs travaux (Saraph, Benson et Schroeder, 1989; Olian et Rynes, 1991; Badri, Davis et Davis, 1995; Bowman, 1998).

\section{Management de la qualité des fournisseurs}

MQF1. L'entreprise n'achète les matières premières qu'auprès des fournisseurs certifiés ISO 9000 (Saraph, Benson et Schroeder, 1989; Badri, Davis et Davis, 1995; Tan et al., 1999).

MQF2. L'entreprise travaille étroitement avec les fournisseurs afin d'améliorer les processus (Saraph, Benson et Schroeder, 1989; Badri, Davis et Davis, 1995; Easton et Jarrell, 1998; Tan et al., 1999; Zhang, Waszink et Wijngaard, 2000).

MQF3. L'entreprise fournit une assistance technique aux fournisseurs (Saraph, Benson et Schroeder, 1989; Badri, Davis et Davis, 1995; Ahire, Golhar et Waller, 1996).

MQF4. L'entreprise a créé un partenariat avec ses fournisseurs (Juran, 1983; Ishikawa, 1984; Saraph, Benson et Schroeder, 1989; Deming, 1991; Powell, 1995; Badri, Davis et Davis, 1995; Ahire, Golhar et Waller, 1996; Legendre et Beaucage, 1997; Grandzol et Gershon, 1998; Bowman, 1998; Easton et Jarrell, 1998; Dean et Yunus, 1999; Zhang, Waszink et Wijngaard, 2000; Najmi et Kehoe, 2000).

\section{Focalisation sur les clients}

FSC1. L'entreprise réalise des études de marché pour déterminer les besoins et désirs des clients (Juran, 1983; Ishikawa, 1984; Deming, 1991; Kelada, 1992; Dean et Bowen, 1994; Mann et Kehoe, 1994; Kelada, 1996; Legendre et Beaucage, 1997; Naveh, Erez et Zonnenshain, 1998; Najmi et Kehoe, 2000; Rahman, 2001).

FSC2. L'entreprise réalise des études pour évaluer la satisfaction des clients (Olian et Rynes, 1991; Kelada, 1992; Mann et Kehoe, 1994; Dean et Bowen, 1994; Ahire, Golhar et Waller, 1996; Kelada, 1996; Legendre et Beaucage, 1997; Handfield, Ghosh et Fawcett, 1998; Easton et Jarrell, 1998; Kannan et al., 1999; Dean et Yunus, 1999; Zhang, Waszink et Wijngaard, 2000; Rahman, 2001; Nilsson, Johnson et Gustafsson, 2001). 
FSC3. Le client est intégré dans le processus de développement des produits (Peters et Waterman, 1983; Olian et Rynes, 1991; Kelada, 1992, 1996; Easton et Jarrell, 1998; Kannan et al., 1999; Zhang, Waszink et Wijngaard, 2000).

FSC4. L'entreprise dispose d'un système de collecte des plaintes des clients (Olian et Rynes, 1991; Mann et Kehoe, 1994; Ahire, Golhar et Waller, 1996; Easton et Jarrell, 1998; Dean et Yunus, 1999; Zhang, Waszink et Wijngaard, 2000; Rahman, 2001).

\section{Soutien continu}

SC1. L'entreprise a mis en place un système de récompense pour encourager les nouvelles idées (Crosby, 1986; Saraph, Benson et Schroeder, 1989; Blackburn et Rosen, 1993; Mann et Kehoe, 1994; Badri, Davis et Davis, 1995; Ahire, Golhar et Waller, 1996;Wruck et Jensen, 1997; Easton et Jarrell, 1998; Deleryd, Deltin et Klefsjo, 1999; Kannan et al., 1999; Zhang, Waszink et Wijngaard, 2000; Rahman, 2001).

SC2. L'organisation insiste sur l'amélioration continue des produits et services (Juran, 1983; Ishikawa, 1984; Deming, 1991; Hackman et Wageman, 1995; Gummer, 1996; Easton et Jarrell, 1998; Rahman, 2001).

SC3. L'engagement continu pour l'amélioration de la qualité est activement manifesté de la part de la direction générale (Juran, 1983; Ishikawa, 1984; Deming, 1991; Hackman et Wageman, 1995; Gummer, 1996; Rahman, 2001).

SC4. La direction générale a nommé un coordinateur qui a la responsabilité de rendre opérationnel le programme qualité au sein de l'entreprise (Glover, 1993 ; Nwabueze et Kanji, 1997 ; Deleryd, Deltin et Klefsjo, 1999).

\section{Amélioration du système qualité}

ASQ1. L'entreprise dispose d'un manuel clair de la qualité (Mann et Kehoe, 1994; Kannan et al., 1999; Zhang, Waszink et Wijngaard, 2000).

ASQ2. Le système qualité dans l'entreprise est amélioré de manière continue (Zhang, Waszink et Wijngaard, 2000). 
ASQ3. L'entreprise dispose d'une procédure de documentation claire (Saraph, Benson et Schroeder, 1989; Mann et Kehoe, 1994; Badri, Davis et Davis, 1995 ; Zhang, Waszink et Wijngaard, 2000).

ASQ4. L'entreprise dispose d'un ensemble d'instructions claires de travail (Zhang, Waszink et Wijngaard, 2000).

\section{Information et analyse}

IEA1. Les informations importantes sont présentées et communiquées aux employés (Saraph, Benson et Schroeder, 1989; Badri, Davis et Davis, 1995; Ahire, Golhar et Waller, 1996).

IEA2. L'entreprise collecte et analyse les données qui sont en relation avec son activité (Hackman et Wageman, 1995; Legendre et Beaucage, 1997; Zhang, Waszink et Wijngaard, 2000; Rahman, 2001).

IEA3. Les informations permettent à l'entreprise d'améliorer ses processus clés, ses produits et ses services (Zhang, Waszink et Wijngaard, 2000).

IEA4. L'entreprise dispose de données précises sur ses concurrents afin d'en dégager des pistes d'amélioration (Ahire, Golhar et Waller, 1996; Legendre et Beaucage, 1997; Zhang, Waszink et Wijngaard, 2000).

\section{Utilisation des techniques statistiques de la qualité}

UTSQ1. Les cartes de contrôle et les graphiques sont utilisés pour mesurer et contrôler la qualité (Juran, 1983; Ishikawa, 1984; Deming, 1991; Dean et Bowen, 1994; Powell, 1995; Ahire, Golhar et Waller, 1996; Easton et Jarrell, 1998; Rahman, 2001).

UTSQ2. La direction générale encourage l'utilisation des méthodes statistiques (Juran, 1983; Ishikawa, 1984; Deming, 1991; Wruck et Jensen, 1997; Grandzol et Gershon, 1998).

UTSQ3. Les employés adhèrent aux programmes de formation aux techniques statistiques de la qualité (Powell, 1995; Ahire, Golhar et Waller, 1996).

UTSQ4. Les techniques statistiques sont utilisées de manière intensive au niveau de notre entreprise (Zhang, Waszink et Wijngaard, 2000).

UTSQ5. Les techniques statistiques sont effectives dans l'amélioration de la qualité des produits (Ahire, Golhar et Waller, 1996). 


\section{Bibliographie}

Ahire, S. L., D.Y. Golhar et M.A. Waller (1996a), «Development and validation of TQM implementation constructs », Decision Sciences, vol. 27, n 1, p. 23-56.

Ahire, S.L. et K.C. O'Shaughnessy (1998), «The role of top management commitment in quality management: an empirical analysis of the auto parts industry », International Journal of Quality Science, vol. 3, n ${ }^{\circ}$ 1, p. 5-37.

Anderson, J.C., M. Rungtusanatham et R.G. Schroeder (1994), «A theory of quality management underlying the Deming management method», Academy of Management Review, juillet.

Anderson, M. et A.S. Sohal (1999), «A study of the relationship between quality management practices and performance in small businesses», International Journal of Quality \& Reliability Management, vol. 16, $\mathrm{n}^{\circ} 9$.

BADRI, M.A., D. DAVIS et D. DAVIS (1995), «A study of measuring the critical factors of quality management», International Journal of Quality \& Reliability Management, vol. 12, n 2, p. 36-53.

Bertram, D. (1991), «Getting started in total quality management», Total Quality Management, vol. 2, no 3, p. 279-282.

BlackBuRn, R. et B. Rosen (1993), «Total quality and human resources management: lessons learned», Academy of Management Executive, vol. 7.

Bowman, J.S. (1998), «Total quality management in Florida: implementation in state agencies », Public Administration Quarterly, vol. 22, $\mathrm{n}^{\circ} 1$.

Chог, T.Y. et K. Евосн (1998), «The TQM paradox: relations among TQM practices, plant performance and customer satisfaction », Journal of Operations Management, vol. 17, p. 59-75.

Crosby, P.B. (1986), La qualité, c'est gratuit, Paris, Economica.

Dale, B.G., R.J. Boaden, M. Wilcox et R.E. Mc Quarter (1997), «Total quality management sustaining audit tool: description and use », Total Quality Management, vol. 8, n ${ }^{\circ}$ 6, p. 395-408.

DAVIS, D. et T.J. FISHER (1994), «The pace of change: a case study of the development of a total quality organization », International Journal of Quality \& Reliability Management, vol. 11, n 8, p. 5-18.

DEAN, J.W. et D.E. Bowen (1994), «Management theory and total quality management: Improving research and practice through theory development », Academy of Management Review, vol. 19, $\mathrm{n}^{\circ}$ 3, p. 392-418.

DEAn, S.E. et K. Yunus (1999), «Small service firms face implementation challenges », Quality Progress, p. 67-75, avril.

Deleryd, M., J. Deltin et B. Klefsjo (1999), «Critical factors for successful implementation of process capability studies », Quality Management Journal, vol. 6, $\mathrm{n}^{\circ} 1, \mathrm{p} .40-59$.

Deming, W.E. (1991), Hors de la crise, Paris, Economica.

Revue internationale P.M.E., vol. 20, nº 2, 2007 
Douglas, T.J. et W.Q. Judge JR (2001), «Total quality management implementation and competitive advantage: the role of structural control and exploration", Academy of Management Journal, vol. 44, p. 158-169.

Dyer, J.H. et W.G. Ouchi (1993), «Japanese-style partnerships - giving company a competitive edge», Sloan Management Review, vol. 35, $\mathrm{n}^{\circ}$ 1, p. 51-63.

EAston, G.S. et S.L. JARRELl (1998), «The effects of total quality management on corporate performance: an empirical investigation», The Journal of Business, vol. $71, \mathrm{n}^{\circ} 2$.

Feigendaum, A.V. (1984), Comment appliquer le contrôle total de la qualité dans votre l'entreprise, Strasbourg, Les dossiers du savoir-faire, Les éditions de l'Entreprise.

Ferrandi, J.M. et P. Valette-Florence (2002), «Premiers test et validation de la transposition d'une échelle de personnalité humaine aux marques », Recherche et applications en marketing, vol. 17, $\mathrm{n}^{\circ} 3, \mathrm{p} .21-40$.

Flynn, B.B., R.G. Schroeder et S. SAKakibara (1994), «A framework for quality management research and an associated measurement instrument», Journal of Operations Management, vol. 11, n 4, p. 339-366.

Fornell, C. et D.F. LARCKer (1981), «Evaluating structural equations models with unobservable variables and measurement error», Journal of Marketing Research, vol. 18, p. 39-50.

ForZA, C. et R. FiLIPPINI (1998), «TQM impact on quality conformance and customer satisfaction: a causal model», International Journal of Production Economics, vol. 55 , p. 1-20.

Glover, J. (1993), «Achieving the organizational change necessary for successful TQM», Asia Pacific Journal of Quality Management, vol. 2, p. 47-64.

Gogue, J.M. (1990), Les six samouraï de la qualité, Paris, Economica, 183 p.

Gonthier, J.M. (1992), «Qualité totale: comment hydro-québec se propose d'y arriver», Revue internationale de gestion, février.

Grandzol, J.R. et M. Gershon (1998), «A survey instrument for standardizing TQM modeling research», International Journal of Quality Science, vol. 3, $\mathrm{n}^{\circ} 1$, p. 80-105.

Gummer, B. (1996), «Total quality management: organizational transformation or passing fancy?», Administration in Social Work.

HACKMAN, J.R. et R. WAGEMAN (1995), «Total quality management: empirical, conceptual, and practical issues», Administrative Science Quarterly, vol. 40, $\mathrm{n}^{\circ} 2$, p. 309-342.

HANDField, R., S. GHosh et S. FAwCETt (1998), «Quality-driven change and its effects on financial performance», Quality Management Journal, vol. 5, n 3, p. 13-30.

IshikAwa, K. (1984), Le TQC ou la qualité à la japonaise, Paris, Afnor, 195 p.

Juran, J.M. (1983), Gestion de la qualité, Paris, Afnor, 517 p.

Revue internationale P.M.E., vol. 20, n 2, 2007 
Kannan, V.R., K.C. TAn, R.B. Handfield et S. Ghosh (1999), «Tools and techniques of quality management : an empirical investigation of their impact on performance », Quality Management Journal, vol. 6, n 3, p. 34-50.

KAYNAK, H. (2003), «The relationship between total quality management practices and their effects on firm performance», Journal of Operations Management, vol. 34, $\mathrm{n}^{\circ}$ 2, p. 1-31.

Kelada, J.N. (1992), Comprendre et réaliser la qualité totale, Dollard-des-Ormeaux, Quafec, $415 \mathrm{p}$.

KeladA, J.N. (1996), Integrating Reengineering with Total Quality, Milwaukee, Wis., ASQC Quality Press, $471 \mathrm{p}$.

KeLly, K. (1992), «Quality: small and midsize companies seize the challenge - not a moment too soon», Business Week, 30 novembre, p. 66-69.

KLine, R.B. (1998), Principles and Practice of Structural Equation Modeling, New York, Guildford Press, $354 \mathrm{p}$.

LaHay, C.W. et J.S. Noble (1998), «A framework for business system and quality management integration», International Journal of Quality \& Reliability Management, vol. 15, $\mathrm{n}^{\circ} 6$.

LAKHal, L. et M. Limam (2000a), «La démarche qualité dans les entreprises tunisiennes: cas des entreprises pilotes », Revue tunisienne des sciences de gestion, vol. $2, \mathrm{n}^{\circ} 2$, p. $95-112$.

LAKHAL, L. et M. LiMAM (2000b), «La relation stratégie-qualité-structure organisationnelle: une recherche empirique auprès des entreprises tunisiennes », Direction et gestion, La revue des sciences de gestion, vol. 35, $\mathrm{n}^{\text {os }} 182-183$, mars-juin, p. 17-25.

Legendre, J.G. et A. Beaucage (1997), Guide d'autoévaluation: version abrégée: le qualimètre: le système de mesure de la qualité au Québec, Mouvement québécois de la qualité, $2^{\mathrm{e}}$ édition, $22 \mathrm{p}$.

Leonard, F.S. et W.E. Sasser (1983), L'art de la qualité, Harvard l'Expansion, printemps.

Mann, R. et D. Kehoe (1994), «An evaluation of the effects of quality improvement on business performance», International Journal of Quality and Reliability Management, vol. 11, $\mathrm{n}^{\circ}$ 4, p. 29-44.

Montaigne, J. et G. Stora (1986), La qualité totale dans l'entreprise, Paris, Les Éditions d'Organisation.

Motwani, J.G., A. Kumar, M.A. Youssef et E. Mahmoud (1997), «Forecasting quality of Indian manufacturing organizations : an exploratory analysis », Total Quality Management, vol. 8, $\mathrm{n}^{\circ}$ 6, p. 361-373.

NAJMi, M. et D.F. Kehoe (2000), «An integrated framework for post-ISO 9000 quality development», International Journal of Quality \& Reliability Management, vol. 17, nº 3, p. 226-258.

Naveh, E., M. Erez et A. Zonnenshain (1998), «Developing a TQM implementation model», Quality Progress, vol. 31, n 2.

Revue internationale P.M.E., vol. 20, nº 2, 2007 
Neuville, J.P. (1996), «La qualité en question», Revue française de gestion, marsavril-mai.

NiLsSOn, L., M.D. Johnson et A. GustafsSon (2001), «The impact of quality practices on customer satisfaction and business results: product versus service organisations », Journal of Quality Management, vol. 6, p. 5-27.

NwabueZe, U. (1996), «Strategic management approach to the implementation of quality management», dans les Actes de l'Annual Meeting of the International Academy of Business Disciplines, Rockeville, Md., 11-14 avril.

Nwabueze, U. et G.K. KANJI (1997), «The implementation of total quality management in the NHS : how to avoid failure», Total Quality Management, vol. 8, $\mathrm{n}^{\circ}$ 5, p. 265-280.

Oberle, J. (1990), «Quality gurus: the men and their message», Training, vol. 28, p. 24-27.

O'LEARY-KeLLY, S.W. et R.J.VOKURKA (1998), «The empirical assessment of construct validity», Journal of Operations Management, vol. 16, p. 387-405.

OliAn, J.D. et S.L. Rynes (1991), « Making total quality work: aligning organizational processes, performance measures, and stakeholders », Human Resource Management, vol. 30, p. 303-333.

Papke-Shields, K.E. et M.K. Malhotra (2001), «Assessing the impact of the manufacturing executive's role on business performance through strategic alignment», Journal of Operations Management, vol. 19, p. 5-22.

Peters, T. et R. WATERMAn (1983), Le prix de l'excellence: Les secrets des meilleures entreprises, Paris, Inter Éditions.

Powell, T.C. (1995), «Total quality management as competitive advantage: a review and empirical study», Strategic Management Journal, vol. 16, p. 15-37.

RAHMAN, S. (2001), «A comparative study of TQM practice and organisational performance of SMEs with and without ISO 9000 certification», International Journal of Quality \& Reliability Management, vol. 18, n 1, p. 35-49.

SARAPh, J.V., P.G. Benson et R.G. Schroeder (1989), «An instrument for measuring the critical factors of quality management», Decision Sciences, vol. 20, $\mathrm{n}^{\circ} 4$, p. $810-892$.

Schaffer, R.H. et H.A. Thomson (1992), «Successful change programs begin with results», Harvard Business Review, p. 80-89.

Sun, H. (2001), «Comparing quality management practices in the manufacturing and service industries: learning opportunities », Quality Management Journal, vol. $8, \mathrm{n}^{\circ} 2$, p. 53-71.

Tамімі, N. (1998), «A second-order factor analysis of critical TQM factors », International Journal of Quality Science, vol. 3, no 1, p. 71-79.

TAN, K.C., V.R. KAnNAN, R.B. HANDFIELD et S. GHosh (1999), «Supply chain management: an empirical study of its impact on performance», International Journal of Operations \& Production Management, vol. 19, n 10, p. 1034-1052.

Revue internationale P.M.E., vol. 20, n 2, 2007 
TAtA, J. et S. Prasad (1998), «Cultural and structural constraints on total quality management implementation», Total Quality Management, vol. 9, $\mathrm{n}^{\circ} 8$.

Thiagarajan, T. et M.ZAIrI (1997), «A review of total quality management in practice: understanding the fundamentals through examples of best practice applications - Part I», Total Quality Management, vol. 9, nº 4 .

Tischler, L. (1996), «Presenting TQM's cultural changes », dans les Actes de l'Annual Meeting of the International Academy of Business Disciplines, Rockville, Md., 11-14 avril.

Weill, M. (2001), Le management de la qualité, Paris, La Découverte.

Wruck, K.H. et M. JENSEN (1997), «Science, specific knowledge and total quality management », Journal of Applied Corporate Finance, vol. 10, n ${ }^{\circ} 2$.

ZHANG, Z., A. WASZINK et J. WIJNGAARD (2000), «An instrument for measuring TQM implementation for Chinese manufacturing companies », International Journal of Quality \& Reliability Management, vol. 17, n 7 . 